\title{
Revascularização endovascular infrainguinal: fatores determinantes para a perviedade
}

\author{
Endovascular infrainguinal revascularization: \\ predictive factors for patency \\ Felipe Nasser ${ }^{1}$, Seleno Glauber de Jesus Silva ${ }^{2}$, Rodrigo Bruno Biagioni ${ }^{2}$, \\ Roberta Cristina de Almeida Campos², Marcelo Calil Burihan², Rhumi Inoguti², \\ Melissa Andreia de Moraes ${ }^{3}$, Orlando da Costa Barros ${ }^{3}$, José Carlos Ingrund ${ }^{4}$, Adnan Neser ${ }^{5}$
}

\section{Resumo}

Contexto: A terapia endovascular tem avançado muito como tratamento para a doença arterial oclusiva infrainguinal, principalmente com o desenvolvimento dos materiais e dos stents autoexpansíveis de nitinol.

Objetivo: Avaliar os resultados e os fatores determinantes da angioplastia fêmoro-poplítea em pacientes portadores de isquemia de membros inferiores.

Métodos: Foram tratados, através de angioplastia com ou sem stent, 114 pacientes, e acompanhados por um período médio de 12 meses. A média de idade foi de 66 anos; $53 \%$ eram do sexo feminino; $23,7 \%$ eram portadores de claudicação incapacitante; $8,8 \%$, de dor isquêmica de repouso; e $67,5 \%$, de lesão trófica. As lesões foram classificadas segundo o TransAtlantic Inter-Society Consensus II em A $(53 \%)$, B $(34 \%)$, C $(5 \%)$ e D $(9 \%)$.

Resultados: A análise angiográfica do leito distal mostrou uma média de $1,4 \pm 1,0$ artérias infrapoplíteas pérvias. $O$ sucesso inicial foi de $97 \%$. No seguimento de 1, 6, 12 e 24 meses, a perviedade primária foi de $94,78,48$ e $31 \%$, e a primária assistida, de $94,84,73$ e $61 \%$, respectivamente $(p=0,005)$. O leito distal pobre e a presença de diabetes melito foram associados a uma menor perviedade primária $(p=0,01)$, enquanto a extensão da lesão não influenciou os resultados. As taxas de salvamento de membro em 6, 12 e 24 meses foram de 95, 90 e $90 \%$, respectivamente.

Conclusões: A extensão das lesões tratadas não foi um fator determinante em nossa casuística para o menor sucesso da angioplastia, o que pode sugerir que as indicações para o tratamento endovascular possam ser ampliadas para pacientes com lesões TransAtlantic Inter-Society Consensus II C/D.

Palavras-chave: Artéria femoral, artéria poplítea, angioplastia com balão, arteriosclerose obliterante.

\begin{abstract}
Background: Endovascular techniques have undergone major advances with regard to the treatment of infrainguinal arterial occlusive disease, mainly as a result of the improvement of new devices and self-expanding nitinol stents.

Objective: To evaluate the results and predictive factors of femoropopliteal angioplasty in patients with critical lower limb ischemia.

Methods: During the study, 114 patients were submitted to angioplasty or stenting and followed during an average of 12 months. Mean age was 66 years, and 53\% were female; $23.7 \%$ presented disabling claudication, $8.8 \%$ ischemic rest pain and $67.5 \%$ tissue loss. Lesions were classified as A $(53 \%)$, B $(34 \%)$, C $(5 \%)$ and D $(9 \%)$ according to the TransAtlantic Inter-Society Consensus II.

Results: Angiographic run-off analysis showed an average of $1.4 \pm 1.0$ patent infrapopliteal artery. Initial success rate was $97 \%$. At $1,6,12$ and 24 months of the follow-up period, primary patencies of $94,78,48$ and $31 \%$, and assisted primary patencies of $94,84,73$ and $61 \%$ were achieved $(\mathrm{p}=0.005)$. Poor run-off and diabetes mellitus were directly associated with lower primary patency rates $(p=0.01)$, while angiographic severity of the lesions did not influence results. Limb salvage rates calculated at 6, 12 and 24 months were 95, 90 and $90 \%$, respectively.
\end{abstract}

Conclusions: Lesion length was not a determinant factor of lower success rates for angioplasty or stenting, which may suggest that indications for endovascular treatment can be extended to patients with TransAtlantic Inter-Society Consensus II C/D lesions.

Keywords: Femoral artery, popliteal artery, balloon angioplasty, arteriosclerosis obliterans.

\footnotetext{
1. Cirurgião vascular assistente, Departamento de Angiorradiologia e Cirurgia Endovascular, Hospital Santa Marcelina (HSM), São Paulo, SP.

2. Cirurgião vascular assistente, Serviço de Cirurgia Vascular, HSM, São Paulo, SP.

3. Cirurgião vascular assistente, Departamento de Ecografia Vascular, HSM, São Paulo, SP.

4. Supervisor, Serviço de Cirurgia Vascular, HSM, São Paulo, SP.

5. Chefe, Serviço de Cirurgia Vascular, HSM, São Paulo, SP.
}

Trabalho apresentado na forma de tema livre especial no 37º Congresso Brasileiro de Angiologia e Cirurgia Vascular, realizado em setembro de 2007, Goiânia, GO.

Não foram declarados conflitos de interesse associados à publicação deste artigo.

Artigo submetido em 14.08.08, aceito em 09.12.08.

$J$ Vasc Bras. 2009;8(1):48-55.

Copyright $@ 2009$ by Sociedade Brasileira de Angiologia e de Cirurgia Vascular 


\section{Introdução}

A doença aterosclerótica oclusiva periférica (DAOP) infrainguinal pode levar a claudicação intermitente ou até mesmo a isquemia crítica com o aparecimento de lesões tróficas. O tratamento primordial para os casos de claudicação intermitente continua sendo o controle dos fatores de risco e os exercícios físicos de caminhada, enquanto a revascularização é reservada para os casos de dor isquêmica de repouso (DIR) ou lesões tróficas ${ }^{1}$. Apesar dos baixos índices de complicação no território infrainguinal, as taxas de perviedade primária da angioplastia transluminal percutânea (ATP), com ou sem o implante de stent, publicados em estudos do início da década, levaram a reservar essa modalidade para pacientes com lesões curtas, alto risco cirúrgico ou que não possuíam conduto autógeno. Entretanto, com o desenvolvimento tecnológico dos materiais de recanalização e dos stents autoexpansíveis de nitinol, a indicação dessa modalidade vem crescendo ao longo dos anos ${ }^{2}$, sendo que a nova publicação do TransAtlantic Inter-Society Consensus (TASC) ${ }^{3}$ já institui a terapia endovascular fêmoro-poplítea como modalidade estabelecida e preferida para os casos de estenoses ou oclusões menores que $10 \mathrm{~cm}$ de extensão.

Os objetivos deste trabalho foram revisar os resultados a curto e médio prazo do tratamento endovascular no segmento fêmoro-poplíteo para pacientes portadores de DAOP e identificar fatores que possam influenciar esses resultados.

\section{Método}

Durante o período de janeiro de 2004 a maio de 2007 (41 meses), foram realizados 116 revascularizações percutâneas no segmento fêmoro-poplíteo em 114 pacientes portadores de isquemia crítica de membros inferiores, configurando uma análise prospectiva de uma série de casos. A indicação do tratamento endovascular foi baseada no exame clínico e avaliação angiográfica. A necessidade urgente de revascularização, a presença de membro inviável, lesões em outros segmentos vasculares que não artérias nativas e insuficiência renal nãodialítica constituíram os critérios de exclusão.

Todos os pacientes tinham registrados seus dados demográficos, comorbidades, categoria clínica segundo as recomendações da Society for Vascular Surgery /International Society for Cardiovascular Surgery (SVS /ISCS $)^{4}$ e anatômica segundo o TASC ${ }^{5}$, assim como seu índice tornozelo-braço (ITB) antes e depois do procedimento. Após o término do período de estudo, todas as lesões foram reclassificadas segundo o TASC II $^{3}$. A classificação do leito distal foi feita pela análise angiográfica e contagem do número de artérias infrapoplíteas pérvias, com formação direta ou indireta do arco plantar, seguindo os parâmetros publicados por Suroviec et al. ${ }^{6}$.

Os procedimentos foram realizados em sala de angiorradiologia com equipamento de subtração digital e contraste não-iônico de baixa osmolaridade. $\mathrm{O}$ acesso vascular foi realizado pela punção anterógrada ou retrógrada contralateral da artéria femoral comum, utilizando bainha $6 \mathrm{~F}$, conforme o nível proximal ou distal da lesão, seguida da heparinização sistêmica endovenosa (5.000 UI in bolus, acrescidos de $1.000 \mathrm{UI} / \mathrm{h}$ após 2 horas de procedimento). Todos os pacientes faziam uso prévio de antiagregantes plaquetários e mantiveram a associação de AAS $200 \mathrm{mg} /$ dia com ticlopidina (500 mg/dia) ou clopidogrel (75 mg/dia) por pelo menos 30 dias, após os quais fizeram uso de apenas um medicamento. Após a transposição da lesão por técnica convencional sob road-mapping, com fios-guias hidrofílicos $0,014 ", 0,018$ " ou 0,035 ", a ATP foi realizada propriamente com cateteres-balão convencionais, compatíveis com a extensão da lesão, utilizando-se pressões de 5 a $15 \mathrm{~atm}$ por 45 segundos. O uso primário ou seletivo de stents foi baseado na localização e extensão da lesão, presença de oclusão, recolhimento elástico (recoil) maior que $30 \%$ ou dissecção da área tratada. Nos casos de longas oclusões, foram utilizados mais de um stent.

Durante o acompanhamento ambulatorial, a vigilância era realizada por meio de exame clínico, medida do ITB, realização de eco-color-Doppler a cada 1, 3, 6, 12,18 e 24 meses e de radiografias de coxa em duas incidências para detecção de fraturas do stent. Caso houvesse indício de piora clínica, com queda de pelo menos uma categoria da classificação da SVS/ISCS, diminuição de pelo menos 0,1 no valor do ITB ou identificação ao eco-color-Doppler de estenose pré, intra, ou pósstent maior que 50\% (caracterizado como um índice sistólico $>2,5)^{7}$, os pacientes eram submetidos a nova 
Tabela 1 - Dados clínicos e demográficos

\begin{tabular}{lc}
\hline & $\mathbf{n}(\mathbf{\%})$ \\
\hline Pacientes & $114(100)$ \\
Masculino & $54(47)$ \\
Feminino & $60(53)$ \\
Idade (anos) & $65,8(100)$ \\
Fatores de risco & $84(74)$ \\
Hipertensão arterial & $66(58)$ \\
Diabetes & $48(42)$ \\
Tabagismo & $10(8,8)$ \\
Quadro clínico & $77(67,5)$ \\
Claudicação & $27(23,7)$ \\
Dor em repouso & \\
Lesão trófica & \\
\hline
\end{tabular}

angiografia e tratamento simultâneo da lesão identificada, seguindo as técnica acima descritas.

$\mathrm{Na}$ análise estatística, empregou-se o método de Kaplan-Meier e Log-Rank para o cálculo de perviedade e sobrevida e da regressão de Cox para definição dos fatores de risco relacionados ao tempo, através do programa SPSS 13.0. Os resultados de perviedade seguiram as recomendações da SVIR para dispositivos de revascularização arterial periférica ${ }^{8}$.

\section{Resultados}

Cento e quatorze pacientes foram submetidos a procedimento de revascularização endovascular fêmoropoplítea ( $53 \%$ do sexo feminino). Os dados demográficos e indicação clínica para a intervenção são mostrados na Tabela 1.

A distribuição dos vasos afetados foi inicialmente classificada segundo o TASC ${ }^{5}$, onde obtivemos os seguintes valores para cada grupo: $A=26 \%, B=29 \%$, $\mathrm{C}=37 \%$ e D $=8 \%$. Em janeiro de 2007, uma nova publicação atualizou a classificação para lesões infrainguinais (TASC II) ${ }^{3}$, ampliando as indicações anatômicas para tratamento endovascular (TASC A e B). Foi feita, então, uma reclassificação de todos os pacientes envolvidos, sendo obtida nova distribuição para cada grupo: $\mathrm{A}=52 \%, \mathrm{~B}=34 \%, \mathrm{C}=5 \%$ e D $=9 \%$, conforme ilustrado na Figura 1.

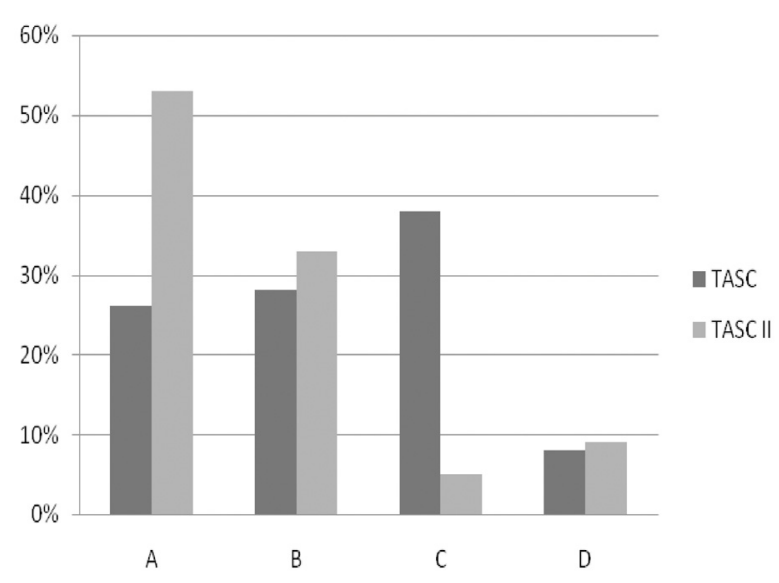

TASC $=$ TransAtlantic Inter-Society Consensus.

Figura 1 - Distribuição percentual dos diferentes grupos de lesões angiográficas conforme a classificação utilizada

O sucesso técnico inicial, considerado como a transposição da lesão, angioplastia e/ou colocação de stent com estenose residual < 30\%, foi obtido em $97 \%$ dos casos. As complicações observadas (dissecção da área tratada, hematoma do sítio de punção, fratura do stent e óbito) são enumeradas na Tabela 2. Nenhum óbito relacionado foi causado diretamente pelo procedimento endovascular, mas por complicações cardiovasculares ou infecciosas relacionadas à lesão trófica. 
Tabela 2 - Complicações pós-tratamento

\begin{tabular}{lc}
\hline Complicação & $\%$ \\
\hline Dissecção da área tratada & 6,0 \\
Hematoma do sítio de punção & 4,3 \\
Fratura do stent & 10,5 \\
Óbito (30 dias) & 4,3 \\
\hline
\end{tabular}

Setenta por cento dos stents utilizados foram autoexpansíveis de nitinol, enquanto que as angioplastias com balão foram realizadas em $15 \%$ dos casos. Os stents balão-expansíveis foram utilizados somente nos primeiros anos do trabalho, sendo que, nos dois últimos anos, somente os stents de nitinol foram empregados.

Os valores de perviedade primária, primária assistida, secundária e salvamento de membro foram obtidos durante o seguimento por 2 anos para cada paciente. Nos intervalos de 1, 6, 12 e 24 meses, a perviedade primária foi de $94,78,48$ e 31\%, e a primária assistida, de 94, 84, 73 e 61\%, respectivamente (Log-Rank; $p<0,05)$, enquanto a perviedade secundária foi de $95,90,83$ e $80 \%$ ( $\mathrm{p}<0,001)$, conforme ilustrado nas Figuras 2 e 3. A taxa de salvamento de membro, considerada como amputação igual ou menor que o nível transmetatársico, possibilitando manter deambulação, em 6, 12 e 24 meses, foi de 95,90 e $90 \%$, respectivamente, conforme Figura 4.

A análise do leito distal revelou uma média de $1,4 \pm 1,0$ artérias infrapoplíteas pérvias, havendo uma distribuição nas seguintes porcentagens: $0=25 \%, 1=35 \%$, $2=23 \%$ e $3=17 \%$. A presença de leito distal adequado (>1 artéria) determinou uma maior perviedade primária em 2 anos ( $p=0,027)$, conforme a Figura 5.

A estatística de regressão de Cox para variáveis vinculadas ao tempo mostrou que apenas a presença de diabetes melito e um leito distal pobre influenciaram negativamente a perviedade primária (Tabela 3 ).

\section{Discussão}

Apesar de o segmento arterial fêmoro-poplíteo constituir mais de 50\% dos casos de DAOP, a decisão sobre o tipo de tratamento ideal permanece complexa. Nos casos de isquemia crítica, o tratamento endovascular tem substituído a revascularização cirúrgica em pacientes de

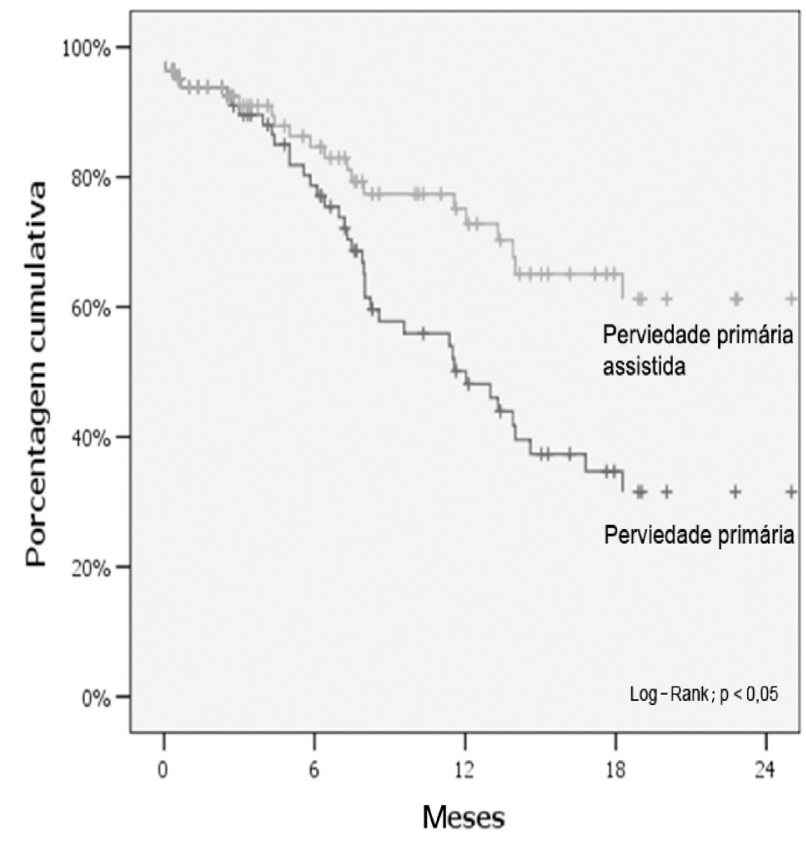

Figura 2 - Análise de perviedades primária e primária assistida no período de 2 anos segundo método de Kaplan-Meier

alto risco cirúrgico, enquanto que, em pacientes com claudicação intermitente, tem sido instituída muitas vezes como associada ao tratamento clínico isolado9.

Há pelo menos uma década, os dados de eficácia e perviedade publicados não eram satisfatórios, em parte devido a características únicas da patologia da artéria femoral superficial ${ }^{9}$, assim como o uso isolado da angioplastia com balão ${ }^{10,11}$ ou de stents de Palmaz balão-expansíveis $^{12,13}$.

A constante evolução tecnológica dos materiais não tem permitido a comparação precisa entre os diferentes grupos de pacientes tratados ao longo dos anos. Entretanto, vários estudos (BLASTER, Mewissen, RESILIENT e SIROCCO I e II ${ }^{14}$ têm demonstrado a 


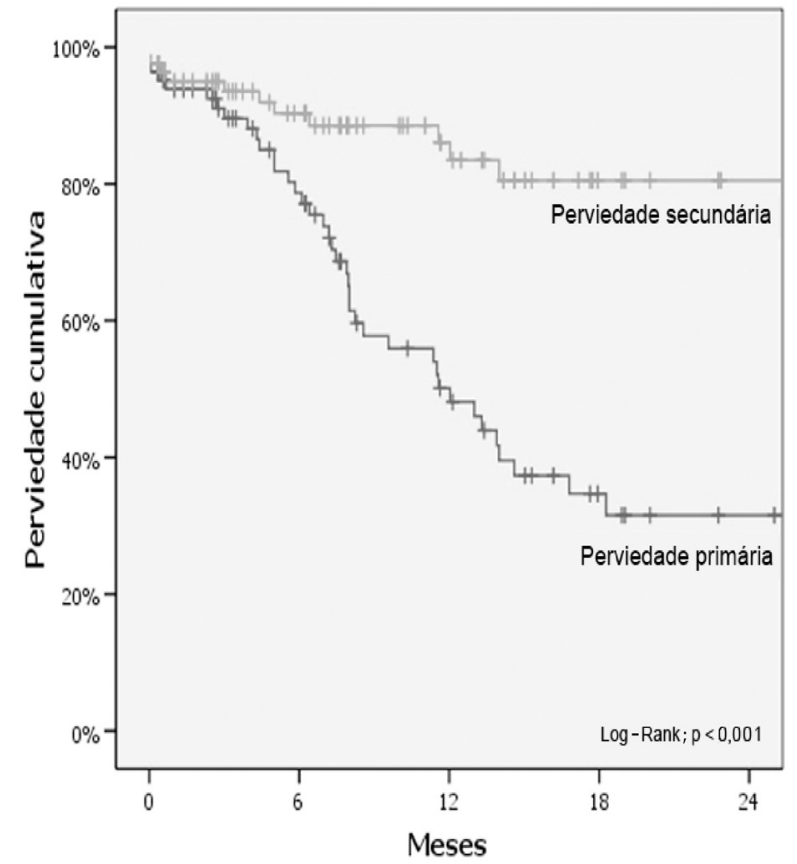

Figura 3 - Análise de perviedades primária e secundária no período de 2 anos segundo método de KaplanMeier

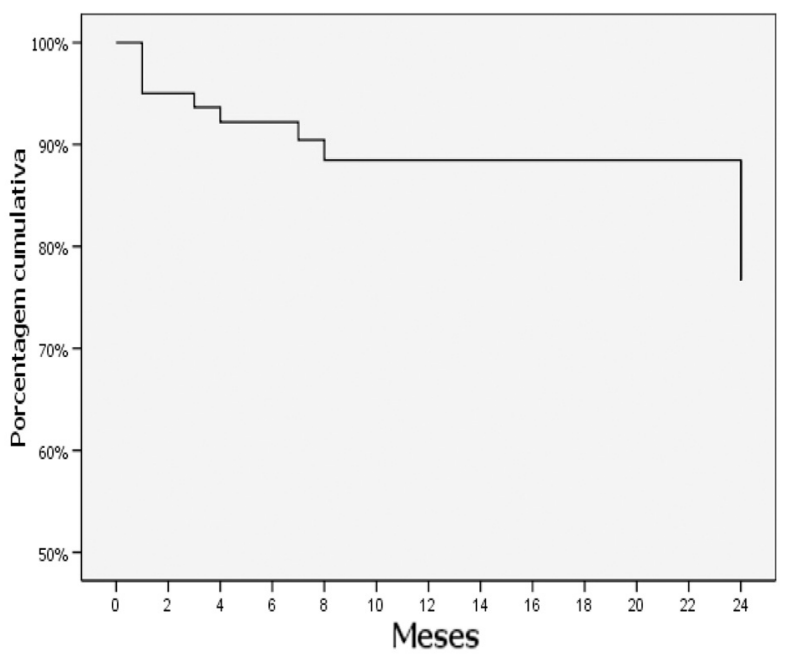

Figura 4 - Tábua de vida para salvamento de membro

superioridade das novas gerações de stent de nitinol. Este avanço vem acompanhado de complicações detectáveis durante o acompanhamento mais longo desses pacientes, como a fratura e a reestenose intra-stent.

Schlager et al. ${ }^{15}$ demonstraram uma taxa de reestenose intra-stent maior com o uso do Wallstent em relação a stents de nitinol e uma grande diferença na taxa

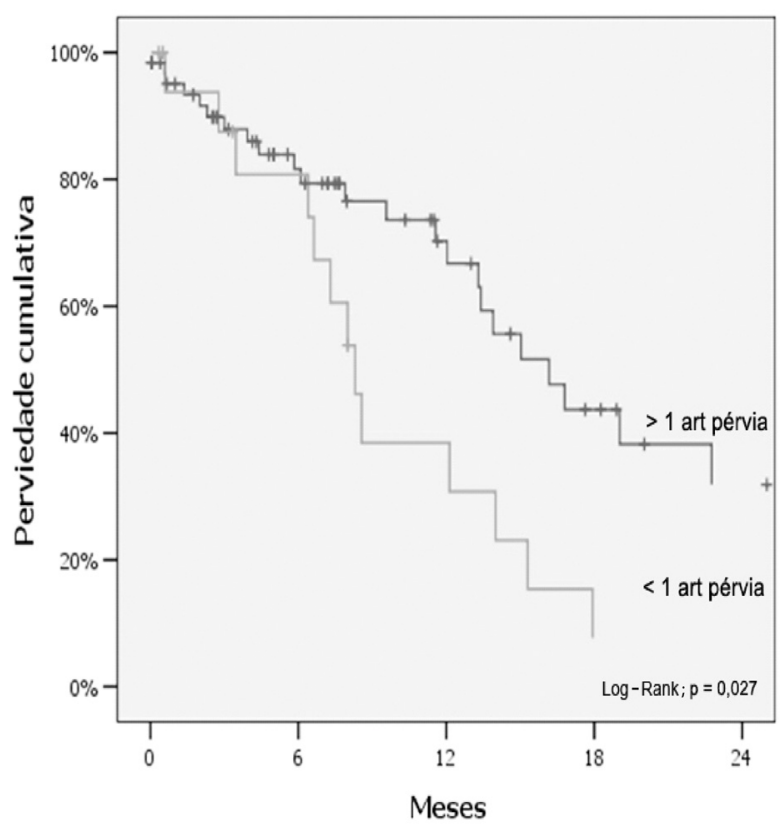

Figura 5 - Perviedade primária segundo análise de KaplanMeier em 24 meses de acordo com o leito distal

de fraturas, mesmo entre os dois grupos de stents de nitinol empregados - SMART (Cordis) e Dynalink /Absolute (Guidant). Por outro lado, o próprio estudo multicêntrico RESILIENT demonstrou, já nos seus primeiros 6 meses de investigação, dados animadores $(2,2 \%$ de fratura, $84,4 \%$ de perviedade primária, com $2 / 3$ de artérias calcificadas e média de 1,8 stents implantados por paciente $)^{16}$. Da mesma forma, Schillinger et al. ${ }^{17,18}$ vêm demonstrando bons resultados com o uso primário de stent autoexpansível em comparação com seu uso seletivo.

Os valores de perviedade primária e primária assistida (Figura 2) em 1 ano obtidos em nosso trabalho (48 e $73 \%$, respectivamente) são mais baixos que os encontrados nos mais recentes trabalhos da literatura (aproximadamente 75 e $85 \%$, conforme a metodologia empregada) $)^{2,3,6,19}$. Isso talvez se deva ao fato de que, nos primeiros anos de estudo, a maioria dos stents disponíveis para nosso uso era pouco flexível (aço e Elgiloy ${ }^{\circledR}$ ), os quais foram progressivamente substituídos pelos de nitinol.

Na tentativa de acompanhar a evolução da indicação do tratamento endovascular no setor fêmoropoplíteo, nós nos propusemos a reclassificar as lesões 
Tabela 3 - Regressão de Cox para perviedade primária

\begin{tabular}{ll}
\hline Fator de risco & IC $<\mathbf{0 , 9 5 \%}$ \\
\hline Idade & 0,473 \\
Sexo & 0,770 \\
HAS & 0,532 \\
DM & 0,011 \\
Tabagismo & 0,151 \\
Quadro clínico & 0,412 \\
Tipo de lesão & 0,532 \\
Leito distal & 0,010 \\
\hline
\end{tabular}

$\mathrm{DM}=$ diabetes melito; $\mathrm{IC}=$ intervalo de confiança; HAS = hipertensão arterial sistêmica .

do presente trabalho para o TASC II (2007), o qual é considerado padrão para o agrupamento de pacientes em diversos ensaios clínicos já publicados. Os dados obtidos (Figura 1) demonstram que a maioria das lesões teve suas indicações ampliadas para as classes A e B (tratamento endovascular de escolha ou preferencial), resultando em um grande número de angioplastias em lesões /oclusões longas, antes consideradas diretamente para a revascularização cirúrgica. Em trabalho retrospectivo recentemente publicado, Ihnat et al. ${ }^{20}$ revisaram casos de angioplastia fêmoro-poplítea utilizando o TASC II e obtiveram resultados semelhantes aos nossos, mesmo para uma amostra de pacientes principalmente constituída de claudicantes.

Diversos trabalhos tentaram relacionar os valores de perviedade das angioplastias com os mais diversos fatores de risco envolvidos. A presença de diabetes melito, leito distal ruim, insuficiência renal, longas lesões oclusivas e isquemia crítica é citada, na literatura, como relacionada à diminuição da perviedade a médio e longo prazo $^{1,2,6,20-23}$, enquanto outros não encontraram uma relação precisa ${ }^{19}$. Nossos achados mostraram que somente o diabetes melito e um leito distal pobre (ausência de artéria infrapoplítea pérvia) influenciaram no resultado em 1 ano de acompanhamento.

Por outro lado, observamos que a extensão das lesões não foi fator determinante para um pior resultado, talvez explicado pelo fato de que a maioria do pacientes de nossa casuística era portadora de isquemia crítica, certamente com lesões extensas e multissegmentares (76\%), contrastando com diversos estudos em que há uma maior taxa de claudicantes, em torno de $60 \%{ }^{2,6,20,24,25}$. Portanto, achamos justificável a realização de angioplastia mesmo naqueles pacientes com lesões mais longas, inclusive classes C e D do TASC II, visto que é possível a manutenção de perviedade e o salvamento de membro (Figura 4) adequado. Isso vale especialmente para os pacientes com morbimortalidade cirúrgica elevada ou ausência de conduto autógeno disponível.

Alguns trabalhos endossam a boa resposta do implante primário de stent, com menos reintervenções e maior perviedade ${ }^{18}$. Achamos que essa tática é eficiente, principalmente em oclusões longas. A perviedade elevada relatada em nossa série, no que concerne a lesões TASC D, pode ser explicada principalmente pela alta taxa de pacientes idosos e debilitados, nos quais tanto a lesão trófica quanto as doenças de base impedem uma plena mobilidade do membro e, por conseguinte, a fratura do stent. Vale ressaltar a baixa incidência de claudicantes no presente trabalho $(23,7 \%)$ em comparação com os trabalhos publicados na literatura (cerca de 60\%).

Percebe-se que o aprimoramento do TASC e a ampliação de sua indicação endovascular refletem as novas técnicas de revascularização percutâneas, os novos materiais disponíveis e os resultados do acompanhamento a médio e longo prazo publicados, geralmente comparáveis à cirurgia convencional. Entretanto, algumas questões levantadas na literatura acerca dos resultados a longo prazo ainda precisam de resposta. As altas 
taxas de oclusão/reestenose e reintervenção das lesões classe D relatadas poderiam ser melhor evitadas com a cirurgia convencional primária? Os pacientes com grande perda tecidual e com leito distal ruim seriam mais bem tratados com amputação primária ou com procedimentos percutâneos de revascularização multissegmentar ${ }^{20}$ ?

Uma possível limitação do nosso estudo é a grande variabilidade da amostra, envolvendo muitas comorbidades associadas a um amplo espectro de quadros clínicos. Além disso, durante os anos, o progresso da tecnologia endovascular disponível no mercado nos impediu de padronizar os materiais para tratamento das lesões oclusivas ateroscleróticas, o que pode ter gerado algum viés nos resultados.

Talvez o grande diferencial dos resultados a médio e longo prazo do tratamento endoluminal seja a vigilância e reintervenção adequadas. O exame clínico seriado, o cálculo do ITB e o eco-color-Doppler, desde que inseridos rotineiramente no acompanhamento desses pacientes, nos permitiram alcançar taxas de perviedades primária assistida e secundária semelhantes às da literatura $^{1,2}$. Esse acompanhamento pós-angioplastia é justificado, uma vez que muitos dos nossos pacientes acabariam, em última instância, sendo submetidos à amputação do membro.

\section{Conclusões}

A angioplastia fêmoro-poplítea pode ser realizada com sucesso inicial e perviedade a curto e médio prazo aceitável, desde que seja instituída uma vigilância e reintervenção adequadas. A ausência de diabetes melito e a presença de pelo menos uma artéria infrapoplítea pérvia foram os fatores que influenciaram um maior sucesso do tratamento. A extensão das lesões tratadas, entretanto, não foi um fator determinante, em nossa casuística, para um menor sucesso da angioplastia, o que pode sugerir que as indicações para o tratamento endovascular possam ser extrapoladas para pacientes com lesões TASC II C/D. Em virtude disso, ficou claro que a nova publicação do TASC consolidou e ampliou definitivamente a indicação do tratamento endovascular no setor fêmoro-poplíteo. Resta aguardar se o futuro do tratamento endovascular das lesões infrainguinais ficará restrito ao implante de stent ou se novas tecnologias (cutting-balloon, crioplastia, endolaser, stents revestidos, stents farmacológicos, etc. $)^{14,26}$ podem ampliar satisfatoriamente nosso leque de opções. Duas publicações recentes levantam essa questão, quando relatam resultados muito melhores que a angioplastia convencional com o uso de stent revestido (Viabhan, Gore) ${ }^{27} \mathrm{e}$ de stent farmacológico (Zilver PTX, Cook) ${ }^{28}$ no tratamento das lesões relativamente curtas da artéria femoral superficial. Certamente, a impossibilidade de executar estudos experimentais e a constante produção de novos materiais nunca nos permitirão definir o tratamento endovascular padrão-ouro para qualquer segmento vascular, mas ajudarão a definir o melhor e menos invasivo tratamento disponível até o momento.

\section{Referências}

1. Black JH 3rd, LaMuraglia GM, Kwolek CJ, Brewster DC, Watkins MT, Cambria RP. Contemporary results of angioplasty-based infrainguinal percutaneous interventions. J Vasc Surg. 2005;425:932-9.

2. Conrad MF, Cambria RP, Stone DH, et al. Intermediate results of percutaneous endovascular therapy of femoropopliteal occlusive disease: A contemporary series. J Vasc Surg. 2006;44:762-9.

3. Norgren L, Hiatt WR, Dormandy JA, et al. Inter-Society Consensus for the Management of Peripheral Arterial Disease (TASC II). J Vasc Surg. 2007;45:S5-67.

4. Rutherford RB, Baker JD, Ernst C, et al. Recommended standards for reports dealing with lower extremity ischemia: Revised version. J Vasc Surg. 1997;26:517-38.

5. Management of peripheral arterial disease (PAD). TransAtlantic Inter-Society Consensus (TASC). Eur J Vasc Endovasc Surg. 2000;19:Suppl A:Si-xxviii, S1-250.

6. Surowiec SM, Davies MG, Eberly SW, et al. Percutaneous angioplasty and stenting of the superficial femoral artery. J Vasc Surg. 2005;41:269-78.

7. Ascher E, Marks NA, Hingorani AP, Schutzer RW, Mutyala M. Duplex-guided endovascular treatment for occlusive and stenotic lesions of the femoral-popliteal arterial segment: a comparative study in the first 253 cases. J Vasc Surg. 2006;44:1230-8.

8. Sacks D, Marinelli DL, Martin LG, Spies JB, Society of Interventional Radiology Technology Assessment Committee. Reporting standards for clinical evaluation of new peripheral arterial revascularization devices. J Vasc Interv Radiol. 2003;14:S395-404.

9. Jaff M. The nature of SFA disease. Endovasc Today. 2004;4:13-15.

10. Golledge J, Ferguson K, Ellis M, et al. Outcome of femoropopliteal angioplasty. Ann Surg. 1999;229:146-53. 
11. Stanley B, Teague B, Raptis S, Taylor DJ, Berce M. Efficacy of balloon angioplasty of the superficial femoral artery and popliteal artery in the relief of leg ischemia. J Vasc Surg. 1996;23:679-85.

12. Cejna M, Thurnher S, Illiasch H, et al. PTA versus Palmaz stent placement in femoropopliteal artery obstructions: a multicenter prospective randomized study. J Vasc Interv Radiol. 2001;12:23-31.

13. Pozzi Mucelli F, Fisicaro M, Calderan L, et al. Percutaneous revascularization of femoropopliteal artery disease: PTA and PTA plus stent. Results after six years' follow-up. Radiol Med. 2003;105:339-49.

14. Laird J. Interventional options in the SFA. Endovasc Today. 2004(Suppl):9-12.

15. Schlager O, Dick P, Sabeti S, et al. Long-segment SFA stenting--the dark sides: in-stent restenosis, clinical deterioration, and stent fractures. J Endovasc Ther. 2005; 12:676-84.

16. Laird JR. The RESILIENT Trial Update: 6-Months Phase 1 Results. Endovasc Today. 2005:29-36. http:// www.evtoday.com/PDFarticles/1005/

EVT1005_John\%20R.\%20Laird,\%20Jr,\%20MD.html

17. Schillinger M, Sabeti S, Loewe C, et al. Balloon angioplasty versus implantation of nitinol stents in the superficial femoral artery. N Engl J Med. 2006;354:1879-88.

18. Schillinger M, Sabeti S, Dick P, et al. Sustained benefit at 2 years of primary femoropopliteal stenting compared with balloon angioplasty with optional stenting. Circulation. 2007;115:2745-9.

19. Vogel TR, Shindelman LE, Nackman GB, Graham AM. Efficacious use of nitinol stents in the femoral and popliteal arteries. J Vasc Surg. 2003;38:1178-84.

20. Ihnat DM, Duong ST, Taylor ZC, et al. Contemporary outcomes after superficial femoral artery angioplasty and stenting: The influence of TASC classification and runoff score. J Vasc Surg. 2008;47:967-74.
21. Clark TW, Groffsky JL, Soulen MC. Predictors of long-term patency after femoropopliteal angioplasty: Results from the STAR registry. J Vasc Interv Radiol. 2001;12:923-33.

22. Sabeti S, Mlekusch W, Amighi J, Minar E, Schillinger M. Primary patency of long-segment self-expanding nitinol stents in the femoropopliteal arteries. J Endovasc Ther. 2005;12:6-12.

23. Dick F, Diehm N, Galimanis A, Husmann M, Schmidli J, Baumgartner I. Surgical or endovascular revascularization in patients with critical limb ischemia: influence of diabetes mellitus on clinical outcome. J Vasc Surg. 2007;45:751-61.

24. Ferreira M, Capotorto LF, Giafar Abuhadba, Marcelo Monteiro, Luiz Lanziotti. Recanalização da artéria femoral superficial com stents Zilver: técnica padronizada e análise retrospectiva de 3 anos. J Vasc Bras. 2006;5:263-70.

25. Krankenberg H, Schlüter M, Steinkamp HJ, et al. Nitinol stent implantation versus percutaneous transluminal angioplasty in superficial femoral artery lesions up to $10 \mathrm{~cm}$ in length: the femoral artery stenting trial (FAST). Circulation. 2007;16:285-92.

26. Leguizamón, JH. Angioplastia de las arterias infrainguinales: cuando el objetivo es preservar la integridad física, anatómica y funcional. Rev Arg Cardiol. 2003;71:157-8.

27. Saxon RR, Dake MD, Volgelzang RL, Katzen BT, Becker GJ. Randomized, multicenter study comparing expanded polytetrafluoroethylene covered endoprosthesis placement with percutaneous transluminal angioplasty in the treatment of superficial femoral artery occlusive disease. J Vasc Interv Radiol. 2008;19:823-32.

28. Endovascular Today News [site na Internet]. Acessado: 24/08/08. Disponível em: http://www.evtoday.com/eNews/ eNews062408.html.

\author{
Correspondência: \\ Felipe Nasser \\ Rua Santa Marcelina, 177 \\ CEP 08270-070 - São Paulo, SP \\ Tel.: (11) 8224.0505
}

